PROCEEDINGS OF THE

AMERICAN MATHEMATICAL SOCIETY

Volume 126, Number 8, August 1998, Pages 2299-2307

S 0002-9939(98)04309-3

\title{
THE PRIMALITY OF SUBFACTORS OF FINITE INDEX IN THE INTERPOLATED FREE GROUP FACTORS
}

\author{
MARIUS B. STEFAN
}

(Communicated by Palle E. T. Jorgensen)

\begin{abstract}
In this paper we prove that any $\mathrm{II}_{1}$-subfactor of finite index in the interpolated free group factor $L\left(F_{t}\right)$ is prime for any $1<t \leq \infty$ i.e., it is not isomorphic to tensor products of $\mathrm{II}_{1}$-factors.
\end{abstract}

\section{INTRODUCTION}

In [8] S. Popa asked the question of whether the $\mathrm{II}_{1}$-factors $L\left(F_{n}\right), 2 \leq n \leq \infty$ are prime i.e., nonisomorphic to tensor products of $\mathrm{II}_{1}$-factors. An example of a prime $\mathrm{II}_{1}$-factor had been given in [7]; this is $L\left(F_{S}\right)$ where $F_{S}$ is a free group with uncountably many generators, suggesting a positive answer. Liming Ge ([3]) has recently solved the problem affirmatively for $2 \leq n<\infty$, using Voiculescu's free entropy $([12],[13])$. We will show in the present paper how Ge's result can be extended to subfactors of finite index in the free group factor $L\left(F_{n}\right)$, for $3 \leq$ $n<\infty, n$ an integer. As a corollary, one obtains that the subfactors of finite index in the interpolated free group factors $L\left(F_{t}\right)$ are prime for $1<t \leq \infty$. In particular, the interpolated free group factors are prime themselves. Recall that the interpolated free group factors were introduced independently by K. Dykema ([1]) and F. Rădulescu ([9]) as a continuation of the discrete series $L\left(F_{n}\right), n \geq 2$.

It is still unknown what are the subfactors of finite index in the free group factors. F. Rădulescu proved in [9] that for $1<N \leq \infty$ and $\lambda^{-1} \in\left\{4 \cos ^{2} \frac{\pi}{n} \mid n \geq 3\right\}$ the interpolated free group factor $L\left(F_{(N-1) \lambda^{-1}+1}\right)$ can be embedded in $L\left(F_{N}\right)$ with finite index $\lambda^{-1}$. In this context, the following question is natural: is it true that the subfactors of finite index in the free group factors are also (interpolated) free group factors? Let us mention here that the free group factors have the Haagerup approximation property $([5])$ as well as all their subalgebras, and this could be the first evidence that the answer to the above question is affirmative. The result obtained in this paper also supports this conjecture.

In obtaining the estimation of the free entropy we benefit from Ge's idea ([3]) of approximating the matrix approximants of the self-adjoint generators by noncommutative polynomials in the elements of a certain Grassmann manifold. We approximate the matrix approximants of the generators by noncommutative polynomials in the elements of some Grassmann manifold and in one more variable

Received by the editors November 27, 1996 and, in revised form, January 10, 1997.

1991 Mathematics Subject Classification. Primary 46L37, 46L50; Secondary 22D25.

Key words and phrases. Free entropy, prime factors.

The author is a member of the Institute of Mathematics, Romanian Academy, Bucharest.

(c)1998 American Mathematical Society 
(matrix). The specific way (Jones' basic construction) in which the subfactor of finite index and the Jones projection generate the factor makes it possible to choose the polynomials to be of first degree in the new variable. We use this fact to generalize Ge's result to subfactors of finite index. Also compare this estimation of the free entropy with the similar ones in [13] and [2].

The main result we get states that if a $\mathrm{II}_{1}$-factor is finitely generated as a von Neumann algebra and if it has a subfactor of finite index that is not prime, then any finite set of at least 3 generators has infinite free entropy. The primality of the subfactors of finite index in the interpolated free group factors is obtained as a corollary, the case $t=\infty$ being treated separately.

\section{The estimation of the free Entropy}

For completeness we first give the definition of the free entropy ([12], [13]). In the following, $M_{k}(\mathbb{C})$ is the set of $k \times k$ complex matrices, $M_{k}^{s a}=M_{k}^{s a}(\mathbb{C})$ is its subset consisting of self-adjoint matrices, and $\tau_{k}$ is the normalized trace on $M_{k}(\mathbb{C})$. If $M$ is a $\mathrm{II}_{1}$-factor with the unique normalized trace $\tau$ and $x_{1}, \ldots, x_{n+p}, n \geq 1, p \geq 0$, are self-adjoint elements of $M$, then one defines first

$$
\begin{gathered}
\Gamma_{R}\left(x_{1}, \ldots, x_{n}: x_{n+1}, \ldots, x_{n+p} ; m, k, \epsilon\right)=\left\{\left(A_{1}, \ldots, A_{n}\right) \in\left(M_{k}^{s a}\right)^{n} \mid\right. \\
\left\|A_{l}\right\| \leq R \forall 1 \leq l \leq n, \exists\left(A_{n+1}, \ldots, A_{n+p}\right) \in\left(M_{k}^{s a}\right)^{p} \text { such that } \\
\left\|A_{n+l}\right\| \leq R \forall 1 \leq l \leq p \text { and }\left|\tau\left(x_{i_{1}} \cdot \ldots \cdot x_{i_{q}}\right)-\tau_{k}\left(A_{i_{1}} \cdot \ldots \cdot A_{i_{q}}\right)\right|<\epsilon \\
\left.\forall i_{j} \in\{1, \ldots, n+p\}, 1 \leq j \leq q, 1 \leq q \leq m\right\}
\end{gathered}
$$

for $R, \epsilon>0$ and $m, k \in \mathbb{N}$, and then, successively,

$$
\begin{aligned}
& \chi_{R}\left(x_{1}, \ldots, x_{n}: x_{n+1}, \ldots, x_{n+p} ; m, k, \epsilon\right) \\
& \quad=\log \left(v_{n} l_{n k^{2}}\left(\Gamma_{R}\left(x_{1}, \ldots, x_{n}: x_{n+1}, \ldots, x_{n+p} ; m, k, \epsilon\right)\right)\right), \\
& \quad \chi_{R}\left(x_{1}, \ldots, x_{n}: x_{n+1}, \ldots, x_{n+p} ; m, \epsilon\right) \\
& \quad=\limsup _{k \rightarrow \infty}\left(\frac{1}{k^{2}} \chi_{R}\left(x_{1}, \ldots, x_{n}: x_{n+1}, \ldots, x_{n+p} ; m, k, \epsilon\right)+\frac{1}{2} n \log k\right), \\
& \quad \chi_{R}\left(x_{1}, \ldots, x_{n}: x_{n+1}, \ldots, x_{n+p}\right) \\
& \quad=\inf \left\{\chi_{R}\left(x_{1}, \ldots, x_{n}: x_{n+1}, \ldots, x_{n+p} ; m, \epsilon\right) \mid m \in \mathbb{N}, \epsilon>0\right\}, \\
& \chi\left(x_{1}, \ldots, x_{n}: x_{n+1}, \ldots, x_{n+p}\right)=\sup _{R>0} \chi_{R}\left(x_{1}, \ldots, x_{n}: x_{n+1}, \ldots, x_{n+p}\right) .
\end{aligned}
$$

The quantity $\chi\left(x_{1}, \ldots, x_{n}: x_{n+1}, \ldots, x_{n+p}\right)$ is the free entropy of $x_{1}, \ldots, x_{n}$ in the presence of $x_{n+1}, \ldots, x_{n+p}$. If $p=0$, then $\chi\left(x_{1}, \ldots, x_{n}: x_{n+1}, \ldots, x_{n+p}\right)$ is simply called the free entropy of $x_{1}, \ldots, x_{n}$ and it is denoted by $\chi\left(x_{1}, \ldots, x_{n}\right)$.

Let $N \subseteq M$ be an inclusion of $\mathrm{II}_{1}$-factors with $[M: N]<\infty$. According to [6] there is a subfactor $P$ of $N$ such that $M=\left\langle N, e_{P}\right\rangle$, where $e_{P}$ is the Jones projection, that is, $e_{P}$ acts on $L^{2}(N, \tau)$ and it is defined by $e_{P}(x \xi)=E_{P}(x) \xi$ for $x \in N$. Here $E_{P}: N \rightarrow P$ is the conditional expectation from $N$ onto $P$ and $\xi$ is the canonical cyclic trace vector in $L^{2}(N, \tau)$. Moreover, operators of the form

$$
a_{0}+\sum_{i=1}^{n} a_{i} e_{P} b_{i}
$$

with $a_{i}, b_{i} \in N$ give a dense $\star$-subalgebra of $M$, and $[M: N]=[N: P]=\tau\left(e_{P}\right)^{-1}$. 
Next we will estimate the free entropy of certain self-adjoint generators of $M$ in the presence of $e_{P}$, some projections that generate $P_{1}$ and $P_{2}$, and certain matrix units, under the hypothesis that $N \simeq P_{1} \otimes P_{2}$.

Suppose that there are polynomials $\phi_{j}^{(0)}=\phi_{j}^{(0) *}, \phi_{j}^{(l)}, \psi_{j}^{(l)}, 1 \leq j \leq n, 1 \leq l \leq n_{j}$, in $\mathbb{C}\left\langle X_{1}, \ldots, X_{r+s}\right\rangle$ (the $\star$-algebra of complex polynomials in $r+s$ self-adjoint noncommutative indeterminates), so that one defines

$$
\begin{aligned}
\phi_{j}\left(X_{1}, \ldots, X_{r+s+1}\right) \\
=\phi_{j}^{(0)}\left(X_{1}, \ldots, X_{r+s}\right) \\
\quad+\sum_{l=1}^{n_{j}}\left(\phi_{j}^{(l)}\left(X_{1}, \ldots, X_{r+s}\right) \cdot X_{r+s+1} \psi_{j}^{(l)}\left(X_{1}, \ldots, X_{r+s}\right)\right. \\
\left.\quad+\psi_{j}^{(l) *}\left(X_{1}, \ldots, X_{r+s}\right) X_{r+s+1} \phi_{j}^{(l) *}\left(X_{1}, \ldots, X_{r+s}\right)\right) .
\end{aligned}
$$

In this situation the following lemma holds.

Lemma. Let $N \subseteq M$ be an inclusion of type $I I_{1}$-factors, and let $R_{1}$ and $R_{2}$ be $I I_{1}$-subfactors of $N$ such that $N=\left\langle R_{1}, R_{2}\right\rangle \simeq R_{1} \otimes R_{2}$.

Let $x_{1}, \ldots, x_{n}, n \geq 1$, be self-adjoint generators of $M$ and let $p_{1}, \ldots, p_{r} \in R_{2}$ and $q_{1}, \ldots, q_{s} \in R_{1}$ be projections, $r, s \geq 1$, such that

$$
\tau\left(p_{1}\right)=\ldots=\tau\left(p_{r}\right)=\tau\left(q_{1}\right)=\ldots=\tau\left(q_{s}\right)=\frac{1}{2}
$$

and

$$
\left\|x_{j}-\phi_{j}\left(p_{1}, \ldots, p_{r}, q_{1}, \ldots, q_{s}, e_{P}\right)\right\|_{2}<\omega, \quad j=1, \ldots, n,
$$

where $\omega$ is a positive number, $\omega<\min \left(\frac{1}{3}, a\right)$. Then

$$
\chi\left(x_{1}, \ldots, x_{n}\right) \leq \frac{n}{2} \log (36 \pi e)+\log \frac{8 a^{2} C}{3}+(n-2) \log \omega
$$

where $a=\max \left\{\left\|x_{j}\right\|_{2}+1 \mid j=1, \ldots, n\right\}$ and $C>0$ is a universal constant.

Proof. Let $k_{0} \geq 1$ be a fixed integer. Then there are von Neumann subalgebras $M_{1}$ and $M_{2}$ of $R_{1}$ and $R_{2}$ respectively, both $\star$-isomorphic to $M_{k_{0}}(\mathbb{C})$. Let $\left\{e_{j l}\right\}_{j, l},\left\{f_{j l}\right\}_{j, l}$ be matrix units for $M_{1}$ and $M_{2}$ respectively. We shall estimate

$$
\chi_{R}\left(x_{1}, \ldots, x_{n}: p_{1}, \ldots, p_{r}, q_{1}, \ldots, q_{s},\left\{e_{j l}\right\}_{j, l},\left\{f_{j l}\right\}_{j, l}, e_{P} ; m, k, \epsilon\right)
$$

for some large $R>0$ (it suffices, though, to assume that $R \leq a$, see [13]), large integers $m, k$ and small $\epsilon>0$.

If $\left(A_{1}, \ldots, A_{n}, G_{1}, \ldots, G_{r}, H_{1}, \ldots, H_{s},\left\{E_{j l}\right\}_{j, l},\left\{F_{j l}\right\}_{j, l}, E\right)$ is an element of $\Gamma_{R}\left(x_{1}, \ldots, x_{n}: p_{1}, \ldots, p_{r}, q_{1}, \ldots, q_{s},\left\{e_{j l}\right\}_{j, l},\left\{f_{j l}\right\}_{j, l}, e_{P} ; m, k, \epsilon\right)$, then

$$
\left\|A_{j}-\phi_{j}\left(G_{1}, \ldots, G_{r}, H_{1}, \ldots, H_{s}, E\right)\right\|_{2}<\omega, j=1, \ldots, n
$$

provided that $m$ is large enough and $\epsilon$ is sufficiently small. Also, for any $\delta>0$ there is an injective $\star$-homomorphism $\alpha_{k}:\left\langle M_{1}, M_{2}\right\rangle \rightarrow M_{k}(\mathbb{C})$ such that

$$
\left\|\alpha_{k}\left(e_{j l}\right)-E_{j l}\right\|_{2}<\delta, \quad\left\|\alpha_{k}\left(f_{j l}\right)-F_{j l}\right\|_{2}<\delta, \quad 1 \leq j, l \leq k_{0},
$$

provided that $m$ is large enough and $\epsilon$ is sufficiently small, but independently of $k$. Eventually, further restricting $m$ and $\epsilon$, we have that $\alpha_{k}\left(e_{j j} f_{l l}\right)$ are projections of trace $\frac{t}{k_{0}^{2} t+w}=\frac{t}{k}$ for $1 \leq j, l \leq k_{0}$, if $\delta$ is small enough and $k=k_{0}^{2} t+w, 0 \leq$ $w \leq k_{0}^{2}-1$. Let $U$ be unitary in $U(k)$ and such that $U \alpha_{k}\left(e_{j j} f_{l l}\right) U^{*}$ are diagonal projections for all $1 \leq j, l \leq k_{0}$. In particular, $U \alpha_{k}(1) U^{*}$ is a diagonal projection 
of trace $1-\frac{w}{k}=\frac{k_{0}^{2} t}{k}$. The commutant of $U \alpha_{k}\left(M_{j}\right) U^{*}$ in $M_{k}(\mathbb{C})$ will then be $\star$-isomorphic to $M_{w}(\mathbb{C}) \oplus M_{k_{0} t}(\mathbb{C})$ for $j=1,2$.

The conditional expectation from $M$ onto $M_{1}^{\prime} \cap M$ is given by

$$
E_{M_{1}^{\prime} \cap M}(x)=\frac{1}{k_{0}} \sum_{j, l=1}^{k_{0}} e_{j l} x e_{l j}=\eta\left(x,\left\{e_{j l}\right\}_{j, l}\right),
$$

and $\eta \in \mathbb{C}\left\langle X_{1}, \ldots, X_{k_{0}^{2}+1}\right\rangle$ is a self-adjoint polynomial in $k_{0}^{2}+1$ self-adjoint indeterminates. With a similar argument we also get that $G_{1}^{\prime}:=\eta\left(G_{1},\left\{\alpha_{k}\left(e_{j l}\right)\right\}_{j, l}\right)$ is in the relative commutant of $\alpha_{k}\left(M_{1}\right)$ in $M_{k}(\mathbb{C})$. From $p_{1}=E_{M_{1}^{\prime} \cap M}\left(p_{1}\right)=$ $\eta\left(p_{1},\left\{e_{j l}\right\}_{j, l}\right)$ we obtain

$$
\left|\tau_{k}\left(G_{1}^{\prime l}\right)-\tau\left(p_{1}^{l}\right)\right|<\delta_{1}, \quad 1 \leq l \leq m_{1},
$$

provided that $\epsilon$ and $\delta$ are small and $m$ is large enough. Using Lemma 4.3 in [12] and the fact that $G_{1}^{\prime} \in \alpha_{k}\left(M_{1}\right)^{\prime} \cap M_{k}(\mathbb{C})$, we get for any $\delta_{2}>0$, if $\delta_{1}$ is small enough and $m_{1}$ is sufficiently large, a projection $P_{1}^{\prime} \in \alpha_{k}\left(M_{1}\right)^{\prime} \cap M_{k}(\mathbb{C})$ such that $\operatorname{rank}\left(P_{1}^{\prime}\right)=\left[\frac{k_{0} t+w}{2}\right]$ and $\left\|P_{1}^{\prime}-G_{1}^{\prime}\right\|_{2}<\delta_{2}$. We have

$$
\begin{aligned}
\left\|G_{1}^{\prime}-G_{1}\right\|_{2}^{2} & =\tau_{k}\left(\left(G_{1}^{\prime}-G_{1}\right)^{*}\left(G_{1}^{\prime}-G_{1}\right)\right)=\tau_{k}\left(\left(G_{1}^{\prime}-G_{1}\right)^{2}\right) \\
& =\tau_{k}\left(\left(G_{1}-\eta\left(G_{1},\left\{\alpha_{k}\left(e_{j l}\right)\right\}_{j, l}\right)\right)^{2}\right) .
\end{aligned}
$$

Also $\tau\left(\left(p_{1}-\eta\left(p_{1},\left\{e_{j l}\right\}_{j, l}\right)\right)^{2}\right)=0$, so that $\left\|G_{1}-G_{1}^{\prime}\right\|_{2}<\delta_{2}$ if $\epsilon, \delta$ are small enough and $m$ is sufficiently large. Hence one obtains

$$
\left\|G_{1}-P_{1}^{\prime}\right\|_{2} \leq\left\|G_{1}-G_{1}^{\prime}\right\|_{2}+\left\|G_{1}^{\prime}-P_{1}^{\prime}\right\|_{2}<2 \delta_{2} .
$$

In the same way we can find projections $P_{2}^{\prime}, \ldots, P_{r}^{\prime} \in \alpha_{k}\left(M_{1}\right)^{\prime} \cap M_{k}(\mathbb{C})$ and $Q_{2}^{\prime}, \ldots, Q_{s}^{\prime} \in \alpha_{k}\left(M_{2}\right)^{\prime} \cap M_{k}(\mathbb{C})$ of rank $\left[\frac{k_{0} t+w}{2}\right]$ such that $\left\|G_{i}-P_{i}^{\prime}\right\|_{2}<2 \delta_{2}$ and $\left\|H_{j}-Q_{j}^{\prime}\right\|_{2}<2 \delta_{2}$ for each $i$ and $j$. If $\delta_{2}$ is small enough, we have, moreover,

$$
\left\|A_{j}-\phi_{j}\left(P_{1}^{\prime}, \ldots, P_{r}^{\prime}, Q_{1}^{\prime}, \ldots, Q_{s}^{\prime}, E\right)\right\|_{2}<\omega, j=1, \ldots, n .
$$

Now, $U P_{1}^{\prime} U^{*}, \ldots, U P_{r}^{\prime} U^{*}$ are in the commutant of $U \alpha_{k}\left(M_{1}\right) U^{*}$ in $M_{k}(\mathbb{C})$, and since this is $\star$-isomorphic to $M_{w}(\mathbb{C}) \oplus M_{k_{0} t}(\mathbb{C}) \subset M_{k_{0} t+w}(\mathbb{C})$ and all these projections are of rank $\left[\frac{k_{0} t+w}{2}\right]$, it follows that $U P_{1}^{\prime} U^{*}, \ldots, U P_{r}^{\prime} U^{*}$ are contained in $G_{1}(k)$, a copy of the Grassmann manifold $G\left(k_{0} t+w,\left[\frac{k_{0} t+w}{2}\right]\right)$. Similarly, $U Q_{1}^{\prime} U^{*}, \ldots, U Q_{s}^{\prime} U^{*}$ are contained in $G_{2}(k)$, another copy of the same Grassmann manifold. Also,

$$
\left\|U A_{j} U^{*}-\phi_{j}\left(U P_{1}^{\prime} U^{*}, \ldots, U P_{r}^{\prime} U^{*}, U Q_{1}^{\prime} U^{*}, \ldots, U Q_{s}^{\prime} U^{*}, U E U^{*}\right)\right\|_{2}<\omega
$$

for $1 \leq j \leq n$. For $\gamma>0$ we can consider a minimal $\gamma$-net $\left\{U_{t}\right\}_{t \in T(k)}$ in $U(k)$ with respect to the operator norm. From [10] we have that $|T(k)| \leq\left(\frac{C}{\gamma}\right)^{k^{2}}$, where $C$ is a universal constant. If one takes $\gamma:=\frac{\omega}{2 a}$, then $\left\|U_{t} A_{j} U_{t}^{*}-U A_{j} U^{*}\right\|_{2} \leq \omega$ and

$$
\left\|U_{t} A_{j} U_{t}^{*}-\phi_{j}\left(U P_{1}^{\prime} U^{*}, \ldots, U P_{r}^{\prime} U^{*}, U Q_{1}^{\prime} U^{*}, \ldots, U Q_{s}^{\prime} U^{*}, U E U^{*}\right)\right\|_{2}<2 \omega
$$

for $1 \leq j \leq n$.

$M_{k_{0} t+w}^{s a}$ can be embedded in $M_{k}^{s a}$ so that the euclidean norm $\|\cdot\|_{e}$ on $M_{k}^{s a}$ induces a $U\left(k_{0} t+w\right)$-invariant metric on the Grassmann manifold

$$
G\left(k_{0} t+w,\left[\frac{k_{0} t+w}{2}\right]\right) .
$$


Let $\rho_{k}$ denote this metric and let $\left\{P_{a}\right\}_{a \in A(k)}$ be a minimal $\theta$-net in the manifold $G\left(k_{0} t+w,\left[\frac{k_{0} t+w}{2}\right]\right)$ with respect to $\rho_{k}$. From [10] we have that $|A(k)| \leq\left(\frac{C h_{k}}{\theta}\right)^{g k}$, where $C$ is a universal constant, $g_{k}=2\left[\frac{k_{0} t+w}{2}\right] \cdot\left(k_{0} t+w-\left[\frac{k_{0} t+w}{2}\right]\right)$ is the dimension of $G\left(k_{0} t+w,\left[\frac{k_{0} t+w}{2}\right]\right)$ and $h_{k} \leq \sqrt{2 k}$ is the diameter of $G\left(k_{0} t+w,\left[\frac{k_{0} t+w}{2}\right]\right)$ in $M_{k}^{s a}$.

Consequently, there are $P_{a_{1}}, \ldots, P_{a_{r+s}} \in G\left(k_{0} t+w,\left[\frac{k_{0} t+w}{2}\right]\right)$ for some $a_{1}, \ldots$, $a_{r+s} \in A(k)$, such that

$$
\left\|P_{a_{i}}-U P_{i}^{\prime} U^{*}\right\|_{e} \leq \theta,\left\|P_{a_{r+j}}-U Q_{j}^{\prime} U^{*}\right\|_{e} \leq \theta, \quad 1 \leq i \leq r, 1 \leq j \leq s .
$$

Since $\phi_{1}, \ldots, \phi_{n}$ are polynomials, there is $D>0$ such that

$$
\begin{aligned}
& \left\|\phi_{j}\left(V_{1}, \ldots, V_{r+s+1}\right)-\phi_{j}\left(W_{1}, \ldots, W_{r+s+1}\right)\right\|_{e} \\
& \quad \leq D\left\|\left(V_{1}, \ldots, V_{r+s+1}\right)-\left(W_{1}, \ldots, W_{r+s+1}\right)\right\|_{e}
\end{aligned}
$$

for any $1 \leq j \leq n$ and any $V_{1}, \ldots, V_{r+s+1}, W_{1}, \ldots, W_{r+s+1} \in\left\{V \in M_{k} \mid\|V\| \leq\right.$ $R$ \}. It follows that

$$
\begin{aligned}
& \left\|U_{t} A_{j} U_{t}^{*}-\phi_{j}\left(P_{a_{1}}, \ldots, P_{a_{r+s}}, U E U^{*}\right)\right\|_{e} \\
& \quad<2 \omega \sqrt{k}+D\left\|\left(U P_{1}^{\prime} U^{*}, \ldots, U Q_{s}^{\prime} U^{*}, U E U^{*}\right)-\left(P_{a_{1}}, \ldots, P_{a_{r+s}}, U E U^{*}\right)\right\|_{e} \\
& \quad \leq 2 \omega \sqrt{k}+D \theta \sqrt{r+s} ;
\end{aligned}
$$

hence $\left\|U_{t} A_{j} U_{t}^{*}-\phi_{j}\left(P_{a_{1}}, \ldots, P_{a_{r+s}}, U E U^{*}\right)\right\|_{e}<3 \omega \sqrt{k}$ if one takes $\theta:=\frac{\omega}{D} \sqrt{\frac{k}{r+s}}$.

So, if we define $F_{a_{1}, \ldots, a_{r+s}}: M_{k}^{s a} \rightarrow\left(M_{k}^{s a}\right)^{n}$ by

$$
F_{a_{1}, \ldots, a_{r+s}}(W):=\left(\phi_{1}\left(P_{a_{1}}, \ldots, P_{a_{r+s}}, W\right), \ldots, \phi_{n}\left(P_{a_{1}}, \ldots, P_{a_{r+s}}, W\right)\right)
$$

then

$$
\operatorname{dist}_{e}\left(\left(A_{1}, \ldots, A_{n}\right),\left(U_{t}^{*}, \ldots, U_{t}^{*}\right)\left(\operatorname{Range}\left(F_{a_{1}, \ldots, a_{r+s}}\right)\right)\left(U_{t}, \ldots, U_{t}\right)\right)<3 \omega \sqrt{n k} \text {. }
$$

Given the particular form of the polynomials $\phi_{j}, F_{a_{1} \ldots, a_{r+s}}$ is an affine function and $d_{k}:=\operatorname{dim}\left(\operatorname{Range}\left(F_{a_{1}, \ldots, a_{r+s}}\right)\right) \leq k^{2}$. The set of all $\left(A_{1}, \ldots, A_{n}\right)$ with the property $(2)$ and $\left\|\left(A_{1}, \ldots, A_{n}\right)\right\|_{e} \leq a \sqrt{n k}$ is contained in the cartesian product of a certain ball of radius $3 \omega \sqrt{n k}+a \sqrt{n k}$ in the affine subspace

$$
\left(U_{t}^{*}, \ldots, U_{t}^{*}\right)\left(\text { Range }\left(F_{a_{1}, \ldots, a_{r+s}}\right)\right)\left(U_{t}, \ldots, U_{t}\right)
$$

of dimension $d_{k}$, and the ball of radius $3 \omega \sqrt{n k}$ in dimension $n k^{2}-d_{k}$. Hence we have the evaluation

$$
\begin{gathered}
\operatorname{vol}_{n k^{2}}\left(\Gamma_{R}\left(x_{1}, \ldots, x_{n}: p_{1}, \ldots, p_{r}, q_{1}, \ldots, q_{s},\left\{e_{j l}\right\}_{j, l},\left\{f_{j l}\right\}_{j, l}, e_{P} ; m, k, \epsilon\right)\right) \\
\leq\left(\left(\frac{C h_{k}}{\theta}\right)^{g_{k}}\right)^{r+s}\left(\frac{C}{\gamma}\right)^{k^{2}} \cdot \operatorname{vol}_{d_{k}}((3 \omega+a) \sqrt{n k}) \cdot v o l_{n k^{2}-d_{k}}(3 \omega \sqrt{n k}) \\
=\left(\frac{C D h_{k}}{\omega} \sqrt{\frac{r+s}{k}}\right)^{(r+s) g_{k}} \\
\cdot\left(\frac{2 a C}{\omega}\right)^{k^{2}} \cdot \frac{(\pi n k)^{\frac{d_{k}}{2}}(3 \omega+a)^{d_{k}}}{\Gamma\left(1+\frac{d_{k}}{2}\right)} \cdot \frac{(\pi n k)^{\frac{n k^{2}-d_{k}}{2}}(3 \omega)^{n k^{2}-d_{k}}}{\Gamma\left(1+\frac{n k^{2}-d_{k}}{2}\right)} .
\end{gathered}
$$


But

$$
\begin{aligned}
h_{k} & \leq \sqrt{2 k}, \quad \omega<\min \left(\frac{1}{3}, a\right), \quad d_{k} \leq k^{2} \\
g_{k} & =2\left[\frac{k_{0} t+w}{2}\right]\left(k_{0} t+w-\left[\frac{k_{0} t+w}{2}\right]\right) \\
& \leq 2 \cdot \frac{k_{0} t+w}{2} \cdot\left(k_{0} t+w-\frac{k_{0} t+w}{2}\right)=\frac{\left(k_{0} t+w\right)^{2}}{2}=\frac{\left(k+k_{0} w-w\right)^{2}}{2 k_{0}^{2}},
\end{aligned}
$$

and

$$
\frac{1}{\Gamma\left(1+\frac{d_{k}}{2}\right)} \cdot \frac{1}{\Gamma\left(1+\frac{n k^{2}-d_{k}}{2}\right)} \leq \frac{2^{\frac{n k^{2}}{2}}}{\Gamma\left(1+\frac{n k^{2}}{2}\right)}
$$

so that

$$
\begin{gathered}
\operatorname{vol}_{n k^{2}}\left(\Gamma_{R}\left(x_{1}, \ldots, x_{n}: p_{1}, \ldots, p_{r}, q_{1}, \ldots, q_{s},\left\{e_{j l}\right\}_{j, l},\left\{f_{j l}\right\}_{j, l}, e_{P} ; m, k, \epsilon\right)\right) \\
\leq\left(\frac{C D \sqrt{2(r+s)}}{\omega}\right)^{\frac{\left(k+k_{0} w-w\right)^{2}}{2 k_{0}^{2}}(r+s)} \cdot\left(\frac{2 a C}{\omega}\right)^{k^{2}} \cdot \frac{2^{\frac{n k^{2}}{2}}(\pi n k)^{\frac{n k^{2}}{2}}(4 a)^{k^{2}}(3 \omega)^{(n-1) k^{2}}}{\Gamma\left(1+\frac{n k^{2}}{2}\right)},
\end{gathered}
$$

since we can assume $D>\frac{\omega}{C \sqrt{2(r+s)}}$. Further,

$$
\begin{aligned}
& \frac{1}{k^{2}} \chi_{R}\left(x_{1}, \ldots, x_{n}: p_{1}, \ldots, p_{r}, q_{1}, \ldots, q_{s},\left\{e_{j l}\right\}_{j, l},\left\{f_{j l}\right\}_{j, l}, e_{P} ; m, k, \epsilon\right)+\frac{n}{2} \log k \\
& \leq \frac{r+s}{2 k_{0}^{2}}\left(1+\frac{k_{0}-1}{k} w\right)^{2} \log \frac{C D \sqrt{2(r+s)}}{\omega}+\log \frac{2 a C}{\omega}+\frac{n}{2} \log 2+\frac{n}{2} \log (\pi n k) \\
& \quad+\log (4 a)+(n-1) \log (3 \omega)-\frac{1}{k^{2}} \log \Gamma\left(1+\frac{n k^{2}}{2}\right)+\frac{n}{2} \log k \\
&= \frac{r+s}{2 k_{0}^{2}}\left(1+\frac{k_{0}-1}{k} w\right)^{2} \log \frac{C D \sqrt{2(r+s)}}{\omega}+n \log k \\
&-\frac{1}{k^{2}} \log \Gamma\left(1+\frac{n k^{2}}{2}\right)+\log \left(8 a^{2} C\right)+(n-2) \log \omega+(n-1) \log 3+\frac{n}{2} \log (2 \pi n)
\end{aligned}
$$

so that, by using Stirling's formula, $\frac{1}{k^{2}} \log \Gamma\left(1+\frac{n k^{2}}{2}\right)=\frac{n}{2} \log \frac{n k^{2}}{2 e}+o(1)$, we get

$$
\begin{aligned}
& \chi_{R}\left(x_{1}, \ldots, x_{n}: p_{1}, \ldots, p_{r}, q_{1}, \ldots, q_{s},\left\{e_{j l}\right\}_{j, l},\left\{f_{j l}\right\}_{j, l}, e_{P} ; m, \epsilon\right) \\
& \leq \frac{r+s}{2 k_{0}^{2}} \log \frac{C D \sqrt{2(r+s)}}{\omega}-\frac{n}{2} \log \frac{n}{2 e}+\frac{n}{2} \log (2 \pi n) \\
& \quad+\log \left(8 a^{2} 3^{n-1} C\right)+(n-2) \log \omega \\
&= \frac{r+s}{2 k_{0}^{2}} \log (C D \sqrt{2(r+s)})+\frac{n}{2} \log (4 \pi e) \\
&+\log \left(8 a^{2} 3^{n-1} C\right)+\left(n-2-\frac{r+s}{2 k_{0}^{2}}\right) \log \omega .
\end{aligned}
$$


Taking $m \rightarrow \infty$ and $\epsilon \searrow 0$, we get, if $R>0$ is large enough,

$$
\begin{aligned}
\chi\left(x_{1}, \ldots, x_{n}: p_{1}, \ldots, p_{r}, q_{1}, \ldots, q_{s},\left\{e_{j l}\right\}_{j, l},\left\{f_{j l}\right\}_{j, l}, e_{P}\right) \\
=\chi_{R}\left(x_{1}, \ldots, x_{n}: p_{1}, \ldots, p_{r}, q_{1}, \ldots, q_{s},\left\{e_{j l}\right\}_{j, l},\left\{f_{j l}\right\}_{j, l}, e_{P}\right) \\
\leq \frac{r+s}{2 k_{0}^{2}} \log (C D \sqrt{2(r+s)})+\frac{n}{2} \log (4 \pi e) \\
\quad+\log \left(8 a^{2} 3^{n-1} C\right)+\left(n-2-\frac{r+s}{2 k_{0}^{2}}\right) \log \omega .
\end{aligned}
$$

But

$$
\chi\left(x_{1}, \ldots, x_{n}\right)=\chi\left(x_{1}, \ldots, x_{n}: p_{1}, \ldots, p_{r}, q_{1}, \ldots, q_{s},\left\{e_{j l}\right\}_{j, l},\left\{f_{j l}\right\}_{j, l}, e_{P}\right)
$$

since $\left\{p_{1}, \ldots, p_{r}, q_{1}, \ldots, q_{s}, e_{P}\right\} \cup\left\{e_{j l}\right\}_{j, l} \cup\left\{f_{j l}\right\}_{j, l} \subset\left\{x_{1}, \ldots, x_{n}\right\}^{\prime \prime}=M$ (see [13]). Hence

$$
\begin{aligned}
\chi\left(x_{1}, \ldots, x_{n}\right) \leq & \frac{r+s}{2 k_{0}^{2}} \log (C D \sqrt{2(r+s)})+\frac{n}{2} \log (4 \pi e) \\
& +\log \left(8 a^{2} 3^{n-1} C\right)+\left(n-2-\frac{r+s}{2 k_{0}^{2}}\right) \log \omega .
\end{aligned}
$$

Since $k_{0}$ is an arbitrary positive integer, the proof is completed.

\section{The MAIN RESUlT}

The next theorem shows that the free entropy is $-\infty$ if the generators can be approximated arbitrarily closely by polynomials as in the lemma.

Theorem. If $N \subset M$ is an inclusion of $I_{1}$-factors with $[M: N]<\infty, x_{1}, \ldots, x_{n}$ are self-adjoint generators of $M, n \geq 3$, and $N \simeq P_{1} \otimes P_{2}$ for some $I I_{1}$-factors $P_{1}, P_{2}$, then

$$
\chi\left(x_{1}, \ldots, x_{n}\right)=-\infty .
$$

Proof. $M=\left\langle N, e_{P}\right\rangle$, and the set $\left\{a_{0}+\sum_{i=1}^{n} a_{i} e_{P} b_{i} \mid a_{i}, b_{i} \in N\right\}$ is a dense $\star-$ subalgebra of $M$. Since $x_{j}=x_{j}^{*}$ for $1 \leq j \leq n$, each $x_{j}$ can be approximated in the $\|\cdot\|_{2}$-norm by elements of the form

$$
a_{0}+\sum_{i=1}^{n}\left(a_{i} e_{P} b_{i}+b_{i}^{*} e_{P} a_{i}^{*}\right)
$$

for some $a_{0}=a_{0}^{*}, a_{i}, b_{i} \in N$. Now, any $\mathrm{II}_{1}$-factor with separable predual is generated by its projections of trace $\frac{1}{2}$ - say $p_{i}$ 's generate $P_{2}$ and $q_{j}$ 's generate $P_{1}$ - so we can find polynomials $\phi_{j}$ as in the lemma such that

$$
\left\|x_{j}-\phi_{j}\left(p_{1}, \ldots, p_{r}, q_{1}, \ldots, q_{s}, e_{P}\right)\right\|_{2}<\omega, \quad 1 \leq j \leq n
$$

for any given $\omega>0$. Taking $\omega \searrow 0$ in (1), we obtain that the free entropy of $\left(x_{1}, \ldots, x_{n}\right)$ is $-\infty$.

Corollary. For any $1<t \leq \infty$ every $I I_{1}$-subfactor $N \subset L\left(F_{t}\right)$ of finite index is prime. In particular the interpolated free group factors are prime for any $1<t \leq \infty$.

Proof. For $n$ an integer, $L\left(F_{n}\right)=\left\langle x_{1}, \ldots, x_{n}\right\rangle$ with $\left(x_{1}, \ldots, x_{n}\right)$ a semicircular system $([11])$. But any finite semicircular system has finite entropy ([12]), so the conclusion holds for any integer $n \geq 3$. 
Now consider $1<t<\infty$ and let $N \subset L\left(F_{t}\right)$ be such that $\left[L\left(F_{t}\right): N\right]<\infty$ and $N \simeq P_{1} \otimes P_{2}$ for some $\mathrm{II}_{1}$-factors $P_{1}$ and $P_{2}$. There is a projection $p=p_{1} \otimes p_{2} \in N$ such that

$$
\tau(p)=\sqrt{\frac{t-1}{[t]+1}} .
$$

Then

$$
p N p \subset p L\left(F_{t}\right) p=L\left(F_{t}\right)_{\tau(p)} \simeq L\left(F_{1+\frac{t-1}{\tau(p)^{2}}}\right)=L\left(F_{[t]+2}\right),
$$

according to the compression formula in [1] and [9]. Moreover, $\left[p L\left(F_{t}\right) p: p N p\right]=$ $\left[L\left(F_{t}\right): N\right]<\infty$ and $p N p \simeq p_{1} P_{1} p_{1} \otimes p_{2} P_{2} p_{2}$, so $L\left(F_{[t]+2}\right)$ would have a finite index $\mathrm{II}_{1}$-subfactor that is not prime, a contradiction.

If $t=\infty$ then $L\left(F_{t}\right)=L\left(F_{\infty}\right)=\left\langle x_{1}, x_{2}, \ldots\right\rangle$ with $\left(x_{1}, x_{2}, \ldots\right)$ an infinite semicircular system. Also if $A_{m}:=\left\langle x_{1}, \ldots, x_{m}\right\rangle$, then $A_{m} \nearrow L\left(F_{\infty}\right)$ and $\left(E_{A_{m}}(x)\right)_{m \geq 1}$ converges in distribution to $x$ for any $x \in L\left(F_{\infty}\right)$. For a fixed integer $n \geq 3$ we can approximate each $x_{j}, 1 \leq j \leq n$, by polynomials in $p_{1}, p_{2}, \ldots, q_{1}, q_{2}, \ldots$ and $e_{P}$ as in the lemma. Since $\chi\left(x_{1}, \ldots, x_{n}\right)$ is finite, one obtains by taking $\omega \searrow 0$ and $k_{0} \rightarrow \infty$ in (3) that

$$
\chi\left(x_{1}, \ldots, x_{n}: p_{1}, \ldots, p_{r}, q_{1}, \ldots, q_{s},\left\{e_{j l}\right\}_{j, l},\left\{f_{j l}\right\}_{j, l}, e_{P}\right)<\chi\left(x_{1}, \ldots, x_{n}\right)
$$

for some projections $p_{1}, \ldots, p_{r} \in P_{2}$ and $q_{1}, \ldots, q_{s} \in P_{1}$, and certain matrix units $\left\{e_{j l}\right\}_{j, l},\left\{f_{j l}\right\}_{j, l}$. Now,

$$
\begin{gathered}
\left(x_{1}, \ldots, x_{n}, E_{A_{m}}\left(p_{1}\right), \ldots, E_{A_{m}}\left(p_{r}\right), E_{A_{m}}\left(q_{1}\right), \ldots, E_{A_{m}}\left(q_{s}\right),\right. \\
\left.\left\{E_{A_{m}}\left(e_{j l}\right)\right\}_{j, l},\left\{E_{A_{m}}\left(f_{j l}\right)\right\}_{j, l}, E_{A_{m}}\left(e_{P}\right)\right)_{m \geq 1}
\end{gathered}
$$

converges in distribution to

$$
\left(x_{1}, \ldots, x_{n}, p_{1}, \ldots, p_{r}, q_{1}, \ldots, q_{s},\left\{e_{j l}\right\}_{j, l},\left\{f_{j l}\right\}_{j, l}, e_{P}\right)
$$

hence $([13])$

$$
\begin{gathered}
\chi\left(x_{1}, \ldots, x_{n}: E_{A_{m}}\left(p_{1}\right), \ldots, E_{A_{m}}\left(p_{r}\right), E_{A_{m}}\left(q_{1}\right), \ldots, E_{A_{m}}\left(q_{s}\right),\right. \\
\left.\left\{E_{A_{m}}\left(e_{j l}\right)\right\}_{j, l},\left\{E_{A_{m}}\left(f_{j l}\right)\right\}_{j, l}, E_{A_{m}}\left(e_{P}\right)\right)<\chi\left(x_{1}, \ldots, x_{n}\right)
\end{gathered}
$$

for some integer $m>n$. Therefore

$$
\begin{aligned}
\chi\left(x_{1}, \ldots, x_{m}\right)=\chi & \left(x_{1}, \ldots, x_{m}: E_{A_{m}}\left(p_{1}\right), \ldots, E_{A_{m}}\left(p_{r}\right), E_{A_{m}}\left(q_{1}\right),\right. \\
& \left.\ldots, E_{A_{m}}\left(q_{s}\right),\left\{E_{A_{m}}\left(e_{j l}\right)\right\}_{j, l},\left\{E_{A_{m}}\left(f_{j l}\right)\right\}_{j, l}, E_{A_{m}}\left(e_{P}\right)\right) \\
\leq \chi & \chi\left(x_{1}, \ldots, x_{n}: E_{A_{m}}\left(p_{1}\right), \ldots, E_{A_{m}}\left(p_{r}\right), E_{A_{m}}\left(q_{1}\right),\right. \\
& \left.\ldots, E_{A_{m}}\left(q_{s}\right),\left\{E_{A_{m}}\left(e_{j l}\right)\right\}_{j, l},\left\{E_{A_{m}}\left(f_{j l}\right)\right\}_{j, l}, E_{A_{m}}\left(e_{P}\right)\right) \\
+ & \chi\left(x_{n+1}, \ldots, x_{m}\right) \\
<\chi & \chi\left(x_{1}, \ldots, x_{n}\right)+\chi\left(x_{n+1}, \ldots, x_{m}\right)=\chi\left(x_{1}, \ldots, x_{m}\right),
\end{aligned}
$$

and this is impossible. We used previously the fact that ([12])

$$
\chi\left(x_{i_{1}}, \ldots, x_{i_{p}}\right)=\chi\left(x_{i_{1}}\right)+\ldots+\chi\left(x_{i_{p}}\right)
$$

for any $1 \leq i_{1}<\ldots<i_{p} \leq m$, if the system $\left(x_{1}, \ldots, x_{m}\right)$ is free. 


\section{ACKNOWLEDGMENT}

I would like to thank F. Rădulescu for his continuous encouragement and many useful discussions.

\section{REFERENCES}

[1] Dykema, K., Interpolated free group factors, Pac. J. Math. 163 (1994), 123-135. MR 95c:46103

[2] Dykema, K., Two applications of free entropy, Math. Ann. 308 (1997), 547-558. CMP 97:15

[3] Ge, L., Applications of free entropy to finite von Neumann algebras, II, Preprint.

[4] Ge, L., Prime Factors, Proc. Nat. Acad. Sci. (USA) 93 (1996), 12762-12763. CMP 97:03

[5] Haagerup, U., An Example of a Non Nuclear $C^{*}$-Algebra which has the Metric Approximation Property, Invent. Math. 50 (1979), 279-293. MR 80j:46094

[6] Jones, V.F.R., Index for Subfactors, Invent. Math. 72 (1983), 1-25. MR 84d:46097

[7] Popa, S., Orthogonal pairs of $\star$-subalgebras in finite von Neumann algebras, J. Op. Theory 9 (1983), 253-268. MR 84h:46077

[8] Popa, S., Free-independent sequences in type $I_{1}$ factors and related problems, Astérisque 232 (1995), 187-202. MR 97b:46080

[9] Rădulescu, F., Random matrices, amalgamated free products and subfactors of the von Neumann algebra of a free group, of noninteger index, Invent. Math. 115 (1994), 347-389. MR 95c: 46102

[10] Szarek, S.J., Nets of Grassmann manifolds and orthogonal group, Proceedings of Research Workshop on Banach Space Theory (Bor-Luh-Lin, ed.), The University of Iowa, June 29-31 (1982), 169-185. MR 85h:58021

[11] Voiculescu, D., Circular and semicircular systems and free product factors. Operator Algebras, Unitary Representations, Enveloping Algebras, and Invariant Theory, Progress in Mathematics 92 (1990), 45-60. MR 92e:46124

[12] Voiculescu, D., The analogues of entropy and of Fisher's information measure in free probability theory, II, Invent. Math. 118 (1994), 411-440. MR 96a:46117

[13] Voiculescu, D., The analogues of entropy and of Fisher's information measure in free probability theory III: the absence of Cartan subalgebras, G.A.F.A. 6, No. 1 (1996), 172-199. MR 96m:46119

Department of Mathematics, University of Iowa, Iowa City, Iowa 52242

E-mail address: stefan@math.uiowa.edu 